\title{
Removal of Particle Bridges From a Porous Material by Ultrasonic Irradiation
}

\author{
PIETRO POESIO and GIJS OOMS* \\ J.M. Burgerscentrum, Delft University of Technology, Laboratory for Aero- \\ and Hydrodynamics, Mekelweg 2, 2628 CD Delft, The Netherlands
}

(Received: 6 December 2005; in final form: 26 January 2006)

\begin{abstract}
Particle bridge formation during the flow of a liquid with particles through a porous material is a fouling mechanism that can block the pores and, hence, decrease the permeability of the material. Ultrasonic irradiation of the material is a cleaning method that can restore the permeability. We make a numerical study of this cleaning method using the lattice-Boltzmann method. We start from a pore blocked by two spherical particles attached to the pore wall by colloidal adhesion forces, thus forming a particle bridge. Next we calculate the hydrodynamic force exerted by a high-frequency acoustic wave on the two particles. By comparing the hydrodynamic force and the adhesion force we investigate, whether the particle bridge will be removed by the ultrasonic irradiation. A sensitivity study is carried out to investigate the influence of some relevant parameters, such as the acoustic wave amplitude, the acoustic frequency, the fluid flow velocity and the ratio of particle diameter and pore diameter. An upscaling procedure is applied to translate the microscopic results for the removal of the particles at the pore level to the permeability improvement of the material at the macroscopic level. A comparison is made between numerical results and experimental data. The agreement is reasonable.
\end{abstract}

Key words: porous material, fluid flow with particles, permeability reduction, ultrasonic cleaning, lattice-Boltzmann method.

\section{Introduction}

During the production of oil from an underground reservoir the permeability of the sandstone in the near wellbore region can decrease dramatically due to fouling of the sandstone by small particles. This attenuation in permeability causes also a decrease in the oil production rate. Several techniques, such as acid treatment or hydraulic fracturing of the near well bore region, have been developed to overcome this problem but several drawbacks make them unattractive. These techniques can be environmental unfriendly, dangerous or expensive. Therefore an environmental friendly and cheap cleaning method has been developed: the irradiation of the near

\footnotetext{
*Author for correspondence: e-mail: g.ooms@wbmt.tudelft.nl
} 
wellbore region by high-frequency acoustic waves. However, during field tests it was found that this technique gave positive results in only about $50 \%$ of the cases, while it failed in the other cases. Therefore, we decided to make a detailed experimental and numerical study of the technique.

It is important to discuss first the mechanisms that cause the permeability reduction by particles. This reduction can be due to bridge formation by particles in the throats of pores inside the sandstone, or due to the deposition of particles onto the pore walls leading to a reduction of the pore diameter. Of course a combination of the two mechanisms is also possible. Which mechanism occurs, depends on production conditions like oil flow rate, particle properties and properties of the sandstone. The cleaning (particle removing) efficiency of ultrasound is different for the two fouling mechanisms. In this publication we assume that the permeability reduction is due to particle bridge formation. The generation of such particles in an oil reservoir may be due to erosion of the reservoir rock by the oil flow through the reservoir during production. Also other causes are possible such as colloidally induced migration of fines. The generation and migration of small particles in a porous material is well described in the book by Khilar and Fogler (1998).

The aim of this publication is to investigate the conditions at which particle bridges can be removed from a porous material by means of highfrequency acoustic waves. We start by developing a numerical model to calculate the hydrodynamic force exerted by a traveling acoustic wave on the particles in a bridge consisting of two particles present at the entrance to a pore throat. Due to computer limitations it is not (yet) possible to make calculations for particle bridges containing more than two particles. We performed the calculations by means of the lattice-Boltzmann method. Also a model is developed to calculate the adhesion force between the two particles in the bridge and the material of the pore walls. Next, we compare the hydrodynamic force and the adhesion force to find out at what conditions a particle will detach from the bridge at the pore throat. We make a sensitivity study by changing the values of some relevant parameters and study their influence on the cleaning effect. Moreover an attempt is made to up-scale the results calculated at the pore level to macroscopic quantities such as the permeability of the porous material. Finally a comparison is made between numerical predictions and experimental data.

It is emphasized, that the work presented in this publication is for Newtonian fluids. Results may be different for non-Newtonian fluids.

\section{Lattice-Boltzmann Method}

\subsection{NUMERICAL TECHNIQUE}

For our numerical simulations the lattice-Boltzmann method is used. The lattice-Boltzmann method uses a mesoscopic model for the fluid behavior, 
which is based on collision rules for the movement of hypothetical particles (not to be confused with the physical particles) on a grid. The grid is a uniform simple cubic lattice. It can be shown that, after averaging, the continuity equation and Navier-Stokes equation are satisfied. The latticeBoltzmann method has been applied earlier to calculate the laminar or turbulent flow in a complex geometry. Our method is based on the work of Eggels and Somers (1995); it is described in detail by Ten Cate (2002). The boundary condition at the surface of a particle is taken into account by means of an induced force-field method, similar to the one used by Derksen and Van den Akker (1999). In this method a particle is represented by a number of points located in its surface. The surface points are evenly distributed with a mutual distance smaller than the grid spacing. The no-slip condition at the particle surface is satisfied in two steps. First, the fluid velocity at each surface point is determined via a first-order interpolation of the fluid velocities in the surrounding grid points. Then, (induced) forces are assumed to be present at the surface points of the particle of such a magnitude that the fluid velocity in the surrounding grid points are changed in such a way that the no-slip condition at the surface points is satisfied. Thereafter, the hydrodynamic drag force $\left(\mathbf{F}_{d}\right)$ and also the torque $\left(\mathbf{M}_{d}\right)$ acting on a particle by the fluid are computed.

The method of induced forces, used to satisfy the no-slip condition at the particle surface, causes a problem in the calculation of the drag force. According to this method the fluid is assumed to be present at all lattice grid points, so also at the locations where the particles are present. The particle presence is only taken into account via the no-slip condition at the surface points of the particles. The (imaginary) fluid inside the particles causes an (unreal) contribution to the drag force on the particles. This problem is well-known and techniques have been developed to correct for the artificial drag force. We do not discuss these techniques here, but refer to the specialized literature mentioned before, see Ten Cate (2002) and references therein.

It is well-known that the lattice-Boltzmann technique can only be applied when the flow is incompressible. This seems in contradiction with our application of the method to acoustic stimulation. However as the acoustic wavelength in our case is much larger than the pore size, the flow may be considered as locally incompressible. This is also well-known from Biot's theory (Biot, 1956).

\subsection{CALIBRATION PROCEDURE FOR THE PARTICLE RADIUS}

As discussed, a particle surface is approximated in the lattice grid by means of particle surface points. It is known that this approximation causes the particle to experience a drag force that corresponds to a particle with a 
diameter larger than the real one (see Ladd, 1994a, b). This effect can be compensated by ascribing to the particle a hydrodynamic radius that is smaller than the real radius. For the determination of this hydrodynamic radius a calibration procedure is applied. A well-known procedure was proposed by Ladd (1994a). He calculated the drag force acting on a particle located in an array of particles with a periodic arrangement in two ways. He applied his lattice-Boltzmann method and used also the analytical solution for this particular problem. From the comparison between the two results he found the hydrodynamic radius. Ladd's calibration procedure was based on a comparison with the analytical solution of Hasimoto (1959) for the drag force $F_{p}$ on a fixed spherical particle in a periodic array of spherical particles by a fluid flow through the array of particles under creeping flow condition. The following expression was derived by Hasimoto

$$
\frac{6 \pi \mu a U_{u}}{F_{p}}=1-1.7601 C_{\tau}^{1 / 3}+C_{\tau}-1.5593 C_{\tau}^{2},
$$

where $\mu$ represents the dynamic viscosity of the fluid, $a$ the particle radius. $C_{\tau}=\frac{4 \pi a^{3}}{3 L^{3}}$ in which $L$ indicates the size of unit cell of the periodic array and $U_{u}$ is the volumetric averaged fluid velocity across the periodic cell. We used the same procedure as Ladd for the determination of the hydrodynamic radius.

As mentioned above the hydrodynamic radius was determined for creeping flow conditions. One may wonder whether this result holds also at finite Reynolds number $(\mathrm{Re})$ when inertia becomes important. In order to investigate this point we also compute with our lattice-Boltzmann code the lift force on a sphere held stationary in a linear shear flow in the presence of a wall at low, but finite, Reynolds number using the hydrodynamic radius as explained above. The numerical calculations are compared with the analytical solution of Cherukat and McLaughlin (1994) in Figure 1. As can be seen the agreement is reasonable.

As the numerical code will be applied to oscillating flow conditions, we check its reliability also for such flow conditions by comparing numerical and analytical results for the drag force on an oscillating spherical particle in an unbounded fluid. The analytical solution for this problem is known; the drag force as function of time is given by

$$
\mathbf{F}=6 \pi \mu a\left(1+\frac{a}{\gamma}\right) \mathbf{u}+3 \pi a^{2} \sqrt{\frac{2 \mu \rho}{\omega}}\left(1+\frac{a}{\gamma}\right) \frac{\mathrm{d} \mathbf{u}}{\mathrm{d} t},
$$

where $\gamma=\sqrt{2 v / \omega}$ can be interpreted as the depth of penetration of rotational flow around the particle. $v$ is the kinematic viscosity of the fluid and $\omega$ the frequency of the oscillation. (Equation. (2) is derived in the book of 


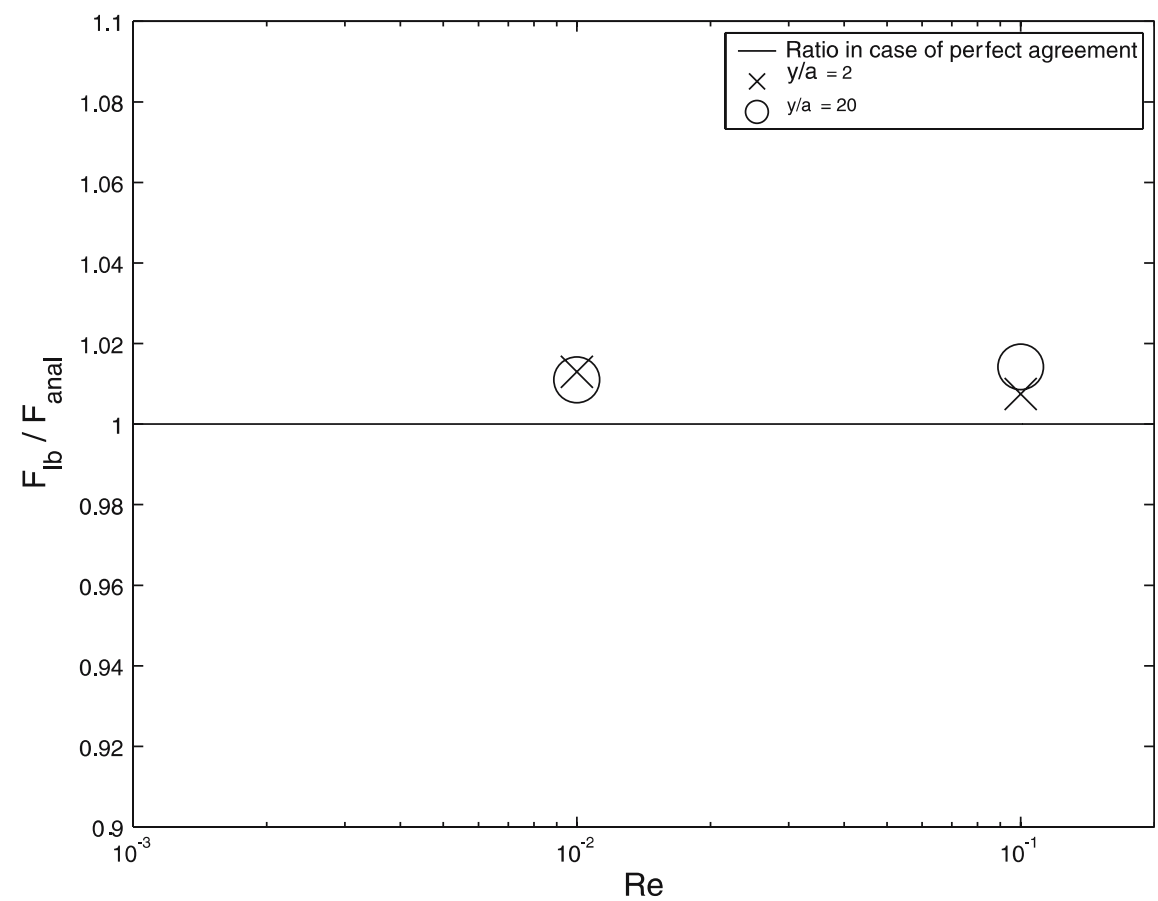

Figure 1. Comparison between the analytically calculated lift force on a particle $F_{\text {anal }}$ and the lift force calculated by our lattice-Boltzmann code $F_{1 \mathrm{~b}}$ for two dimensionless distances from the wall $(y / a=2$ and $y / a=20)$.

Landau and Lifshitz (1959). It is stated that in the limit of $\omega=0$ the equation becomes Stokes' formula, while for high frequencies $\mathbf{F}$ contains a dissipative force and an inertial force.) We computed the maximum drag force during an oscillation cycle and compared it with the theoretical expression given by (Equation (2)). The results are shown in Figure 2. As can be seen, the drag force is reliably predicted by the lattice-Boltzmann code.

\subsection{GEOMETRY OF THE FLOW DOMAIN}

The computations are carried out on a three-dimensional grid with a geometry as sketched in Figure 3. As can be seen this geometry is used to simulate the blocking of a cylindrical pore by two particles. The particles are assumed to have their centers in a plane passing through the pore axis. Since the geometry is three-dimensional, the fluid can go around the particles. So complete blocking is not possible in a three-dimensional flow. We choose the radius of the particles to be equal to 10 lattice units. The radius of the wide part of the pore channel is chosen in such a way that any further enlargement does not change the results. Also the lengths of 


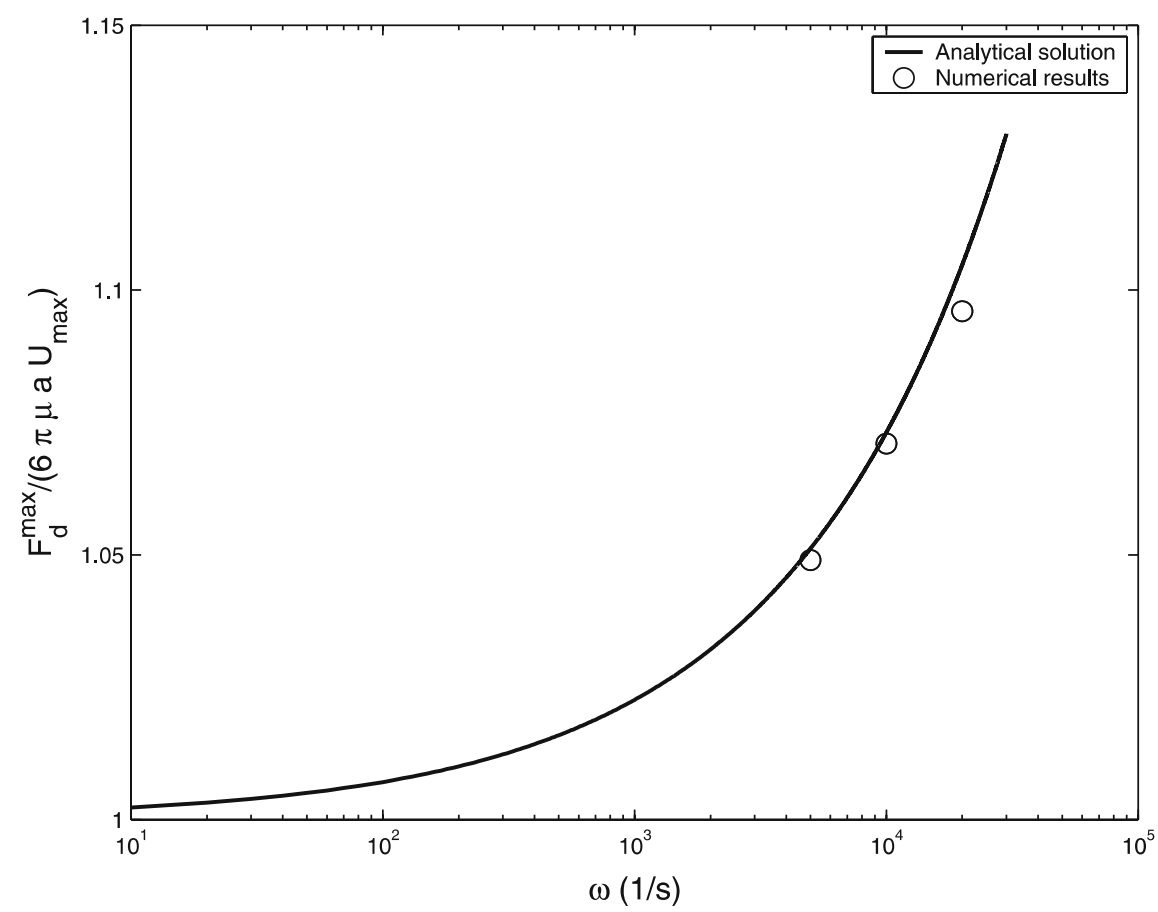

Figure 2. Comparison between the analytical and numerical results for the drag force in a unbounded oscillating flow.
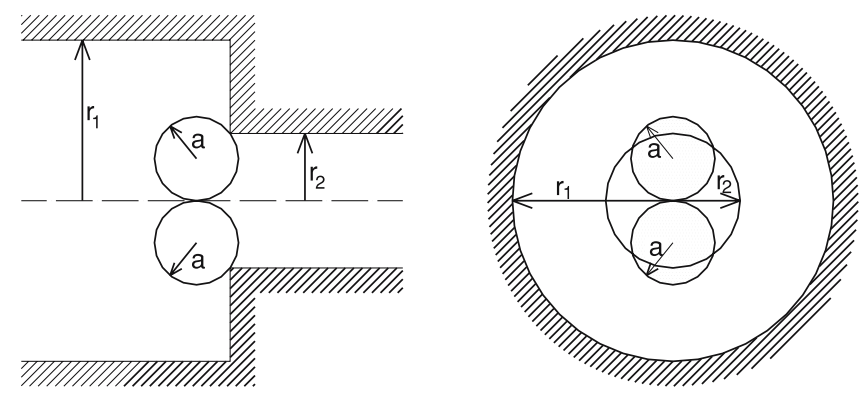

Figure 3. Geometry of the flow domain. Two particles are shown partially blocking the entrance to a pore.

the wide and narrow part of the pore are chosen in such a way that the results do not change with a further increase in length of those parts. The diameter of the narrow part of the pore is a parameter in our calculations. A parabolic inflow and outflow profile of the fluid is imposed. This condition is justified since we study acoustic frequencies well below the critical Biot frequency (Biot, 1956). All the walls are non-slip boundary conditions. 
For some cases we double the number of mesh points in each direction to check whether the numerical results are independent of the mesh size.

\section{Modeling of the High-frequency Acoustic Wave}

As discussed we are interested in the possibility of removal of a (two particle) bridge structure from a pore throat (see Figure 3) by a high-frequency acoustic wave. To that purpose we will calculate the drag force on the particles in the bridge structure due to the oscillating flow generated by the ultrasonic wave. The pore diameter is of the order of a few micrometers, while the acoustic wave length is of the order of a few centimeters. So (as mentioned earlier) the flow can be considered incompressible. The acoustic wave is modeled in the numerical simulation by adding an oscillating body force of the following form

$$
\mathbf{f}=\rho \omega A \sin \omega t \mathbf{i}_{x}
$$

where $\rho$ is the fluid density and $A$ the acoustic wave amplitude. $\mathbf{i}_{x}$ is the unit vector in the direction of wave propagation.

We will compare numerical predictions made with the lattice-Boltzmann code for acoustic cleaning at the pore level with results from acoustic cleaning experiments carried out on sandstone cores in the laboratory. In order to be able to make such a comparison it is necessary to know the amplitude $A$ of the oscillating body force as applied during the experiments with a core and use it in the numerical simulations at the pore level. This amplitude is calculated by means of the following relation for the oscillating pressure

$$
\frac{\mathrm{d} p}{\mathrm{~d} l}=\frac{P}{S} \rho e^{i \omega t},
$$

in which $p$ is the hydrodynamic pressure generated by the acoustic source in the pore liquid during the ultrasonic cleaning experiments with sandstone cores, $l$ the coordinate in the direction of the centerline of the core, $P$ the acoustic power input during the experiments, $S$ the irradiated surface area, $\rho$ the fluid density and $t$ the time. So when $P$ and $S$ are known, it is possible to determine the amplitude of the oscillating pressure in the fluid and also the amplitude $A$ of the body force. This equation is only valid in the very first part of the core close to the acoustic source, where damping of the oscillating wave is still negligible. At larger distances from the source damping has to be taken into account. So at distance $x_{0}$ from the acoustic source the pressure gradient is given by

$$
\left(\frac{\mathrm{d} p}{\mathrm{~d} l}\right)_{x_{0}}=\frac{P}{S} \rho e^{i \omega t-\beta x_{0}}
$$


where $\beta$ is the damping coefficient of Biot's fast wave, see Biot (1956).

The reason for only taking into account the fast wave is that we are interested in the effect of acoustic stimulation over a distance of about $30 \mathrm{~cm}$. As Biot's slow wave is damped very quickly (in about $2 \mathrm{~mm}$ ) we do not need to consider it here. Shear waves are not present because of the perpendicular incidence of the acoustic waves on the surface of the sandstone in our experiments. So we only consider Biot's fast wave. Therefore in Equation (5) $P$ is the acoustic power of the fast wave. As Biot's theory does not reliably predicts the damping coefficient of the fast wave, we use the measured damping coefficient reported by Poesio, Ooms, Barake and Van der Bas (2002), Poesio, Ooms, Schraven and Van der Bas (2002) and Kelder (1998).

Next to the oscillating flow in the sandstone core due to the acoustic wave, there is a steady flow through it as well because of the net oil flow rate during production. This steady component is also taken into account in our numerical simulations. The Reynolds number for the steady flow component based on the pore radius is between $10^{-4}$ and $10^{-3}$.

In our simulations the aspect ratio (ratio of pore diameter and particle diameter) is between 1 and 2 . In real conditions the aspect ratio can be of the order of 20 and particle bridges can be formed consisting of many particles. Because of computer limitations we will restrict ourselves to a bridge consisting of two particles.

\section{Modeling of the Adhesion Force Between a Particle and the Pore Throat Material}

As discussed by Poesio, Ooms, Van Dongen and Smeulders (2004) the adhesion force acting on a particle in the two-particles bridge is due to the attractive van der Waals force between the particle and the pore throat material. For symmetry reason this force lies in the plane through the centerline of the pore and the particle centers. To compute the adhesion force we use the following method: we assume the material of the pore throat walls to be composed of fictitious small particles of radius $\delta_{i}$ and we compute the force acting between the (real) particle in the particle bridge and each fictitious particle and sum all the contributions. The force between the real particle and a fictitious particle is given by

$$
F_{v d W}\left(a, \delta_{i}, x_{i}\right)=-\frac{H}{6}\left(\frac{2 a \delta_{i}}{x_{i}^{2}-\left(a+\delta_{i}\right)^{2}}+\frac{2 a \delta_{i}}{x_{i}^{2}-\left(a-\delta_{i}\right)^{2}}+\ln \frac{x_{i}^{2}-\left(a+\delta_{i}\right)^{2}}{x_{i}^{2}-\left(a-\delta_{i}\right)^{2}}\right),
$$

where $H$ is the Hamaker constant, $x_{i}$ is the distance between the center of the fictitious particle $i$ and the real particle and $\delta_{i}$ is the radius of the 


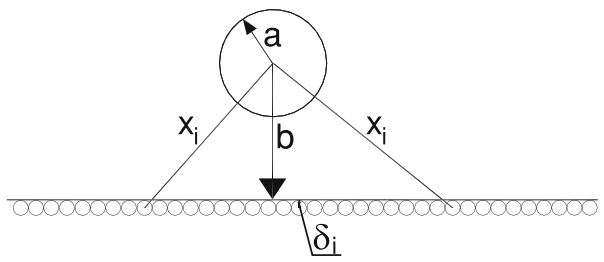

Figure 4. Sketch of the model used for the calculation of the adhesion force. The material of the wall is assumed to be composed of many fictitious particles and their interaction with the real particle is calculated and summed.

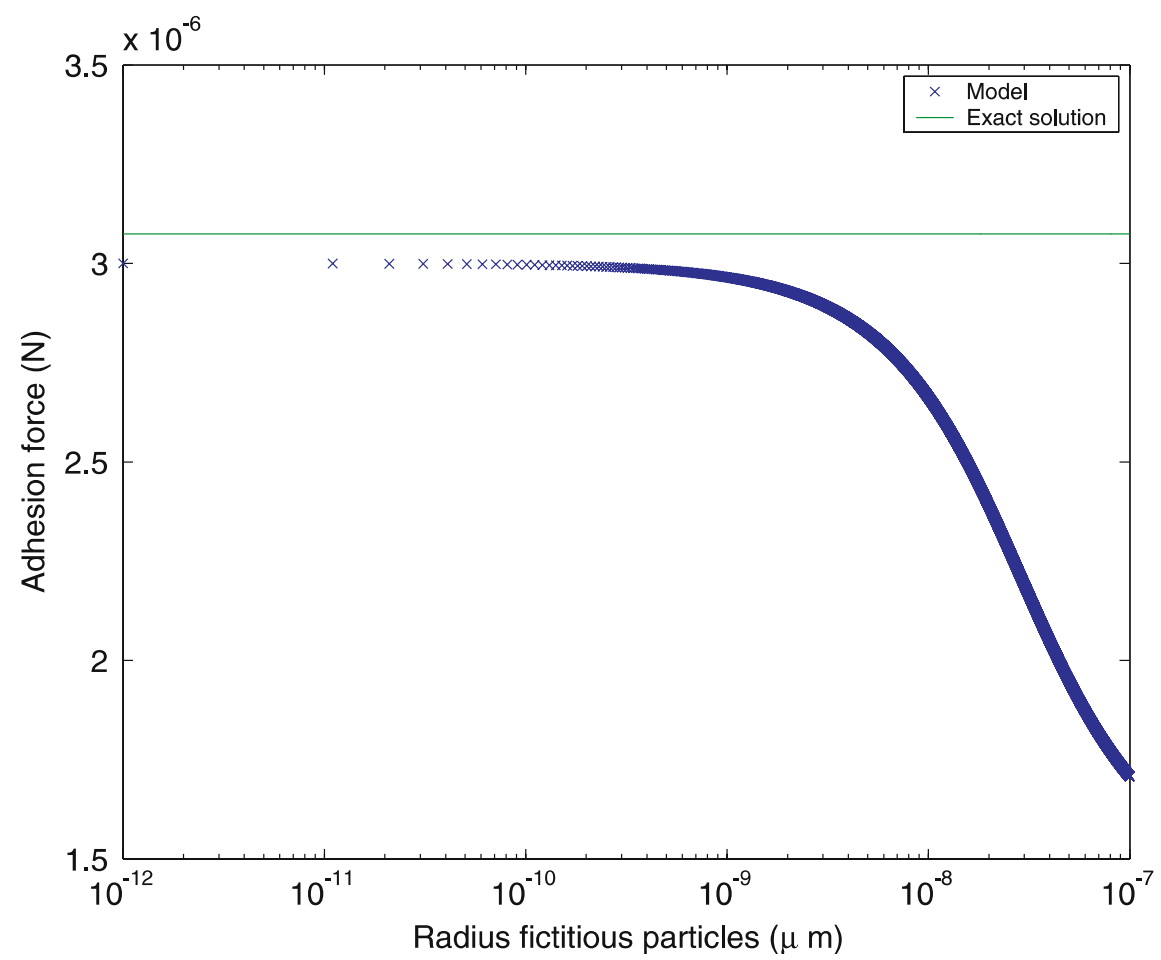

Figure 5. Comparison between numerical result and analytical solution for the particle-wall adhesion force as function of the fictitious particle radius $\delta_{i}$ for the geometry shown in Figure 4.

fictitious particles. The Hamaker constant is dependent upon the material properties only.

To verify the validity of this calculation method, we compare its predicted adhesion force with the known analytical expression for a spherical particle interacting with a plane wall at a distance $b$ of the wall (see Figure 4). When the fictitious particles become small enough we expect that the calculated adhesion force becomes independent of the radius $\delta_{i}$ of the fictitious particles and equal to the analytical expression. The result is 


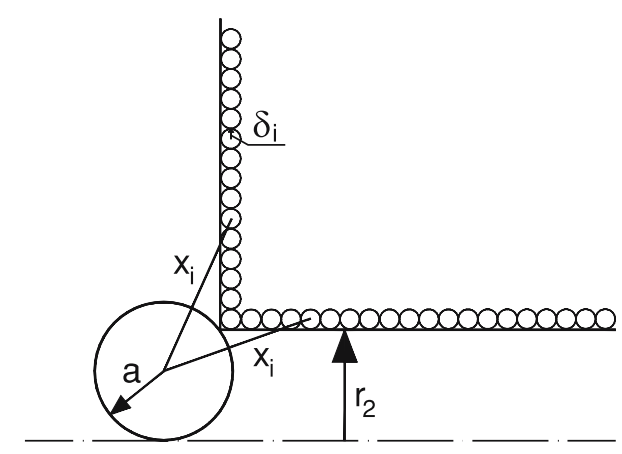

Figure 6. Sketch of the model used for the calculation of the adhesion force between a particle and the pore throat material.

given in Figure 5. Indeed for small enough fictitious particles the numerical adhesion force becomes independent of $\delta_{i}$ and approximates the analytical solution (although the difference remains about 7\%). A possible explanation of the difference between the exact solution and our simplified model is that we use only one layer of fictitious particles. Perhaps the agreement improves, when more layers are considered. However, we think that the approximation by one layer is sufficiently accurate for our purposes in this publication.

In the same way we calculate the adhesion force for the geometry as given in Figure 6: a particle at the entrance of a pore throat. The calculated (horizontal and vertical) components of the force are given in Figure 7. Again when the radius of the fictitious particles is small enough the calculated result is independent of $\delta_{i}$. During these calculations we also find, that the adhesion force is independent of the lengths of the wide and narrow part of the pore when these lengths are larger than twice the particle radius. This effect can be easily understood since the adhesion force is decaying very rapidly with the distance $x_{i}\left(\sim 1 / x_{i}^{2}\right)$. For the geometry of Figure 6 we find that the adhesion force is of the order of $10^{-8} \mathrm{~N}$; two orders of magnitude smaller than the adhesion force for the geometry of Figure 4. This result can explain why, for a given acoustic power, it is much easier to remove a particle bridge structure from a pore throat than a particle adhering to a pore wall (Poesio, Ooms, Van Dongen and Smeulders, 2004).

\section{Numerical Results}

\subsection{INTRODUCTION}

In this section we study the cleaning by ultrasonic waves in detail. We do this by comparing the numerical results for the hydrodynamic force acting 


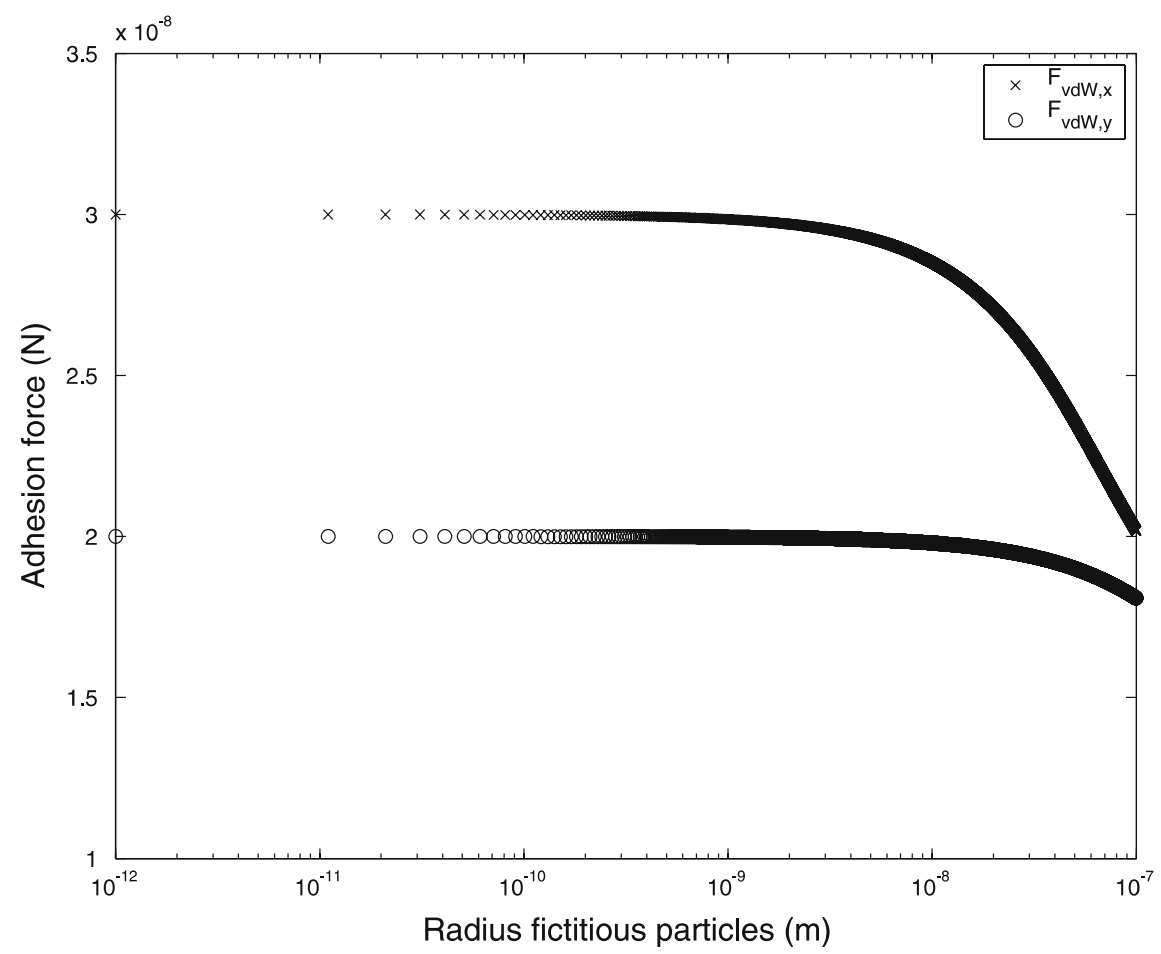

Figure 7. Calculated horizontal $F_{v d W, x}$ and vertical $F_{v d W, y}$ component of the adhesion force as function of the fictitious particle radius $\delta_{i}$ for the geometry given in Figure 6.

on the particles in a (two-particles) bridge (during the negative part of the wave when the particles are pushed away from the pore throat) with the adhesion force acting on these particles because of their interaction with the pore wall material. We will pay particular attention to the influence of different parameters such as the acoustic wave amplitude, the steady fluid flow rate, the aspect ratio and acoustic frequency. The results are expressed in terms of the ratio between the hydrodynamic force and the adhesion force as function of the different parameters. We assume that the particle bridge is broken when this ratio is above unity. It is possible that, in reality, a new bridge is formed during the subsequent positive part of the acoustic wave when the particles are again pushed in the direction of the pore throat. However, that possibility is not included in our calculations.

\subsection{CRITERIA FOR PARTICLES BRIDGE REMOVAL}

Removal of a particle bridge can occur in two ways: either the bridge structure is removed as a whole from the throat or the bridge is broken due 


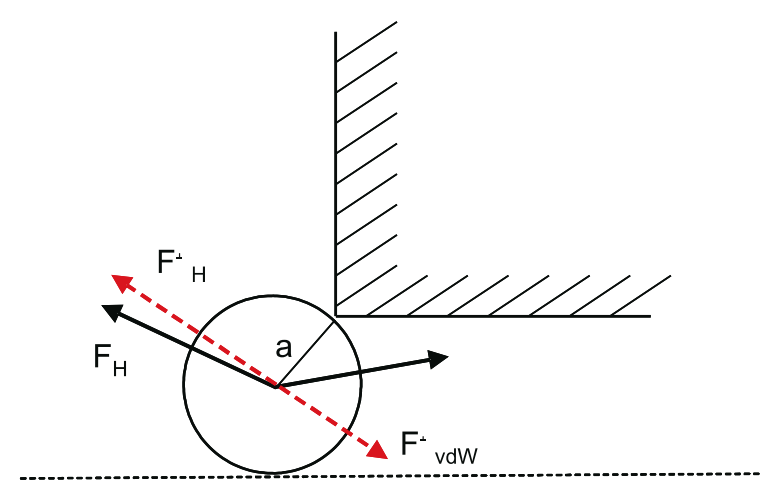

Figure 8. Sketch to explain the calculation procedure for the torque on a particle.

to rotation of the particles around the edge of the pore throat. In the first case the maximum drag force acting on the particles has to overcome the adhesion force in the direction of the pore axis. In the second case the torque (due to the hydrodynamic force) acting on a particle has to be larger than the adhesion torque acting on the particle. It turns out, that particle bridge removal due to rotation of the particles around the pore throat edge is easier than removal of the bridge structure as a whole. To compute the torque it is assumed that the pivotal point is the pore edge (see Figure 8). The torque is then found by the (vector) multiplication of the component of the hydrodynamic force (and the adhesion force) perpendicular to the radius joining the particle centre to the pivotal point and that radius.

\subsection{INFLUENCE OF ACOUSTIC WAVE AMPLITUDE}

The magnitude of the detaching hydrodynamic force increases as the acoustic wave amplitude (so the acoustic power input) increases. As the adhesion force is constant, it can be expected that particle bridge removal will only take place above a certain value of the acoustic power input. This effect is shown in Figure 9, in which the ratio between the hydrodynamic force and the adhesion force is given as function of the ratio $\left(U_{A}-U_{S}\right) / U_{A}$ for two values of the aspect ratio. $U_{A}$ is the maximum fluid velocity generated by the acoustic wave and $U_{S}$ is the steady fluid flow velocity (representing the oil flow rate through a reservoir during production). $U_{A}$ is, of course, directly related to the acoustic wave amplitude and frequency. As expected particle bridge removal can only occur, when the fluid flow velocity $\left(U_{A}\right)$ generated by the acoustic wave is sufficiently large. Hence when the acoustic wave amplitude or the acoustic power input is sufficiently large. 


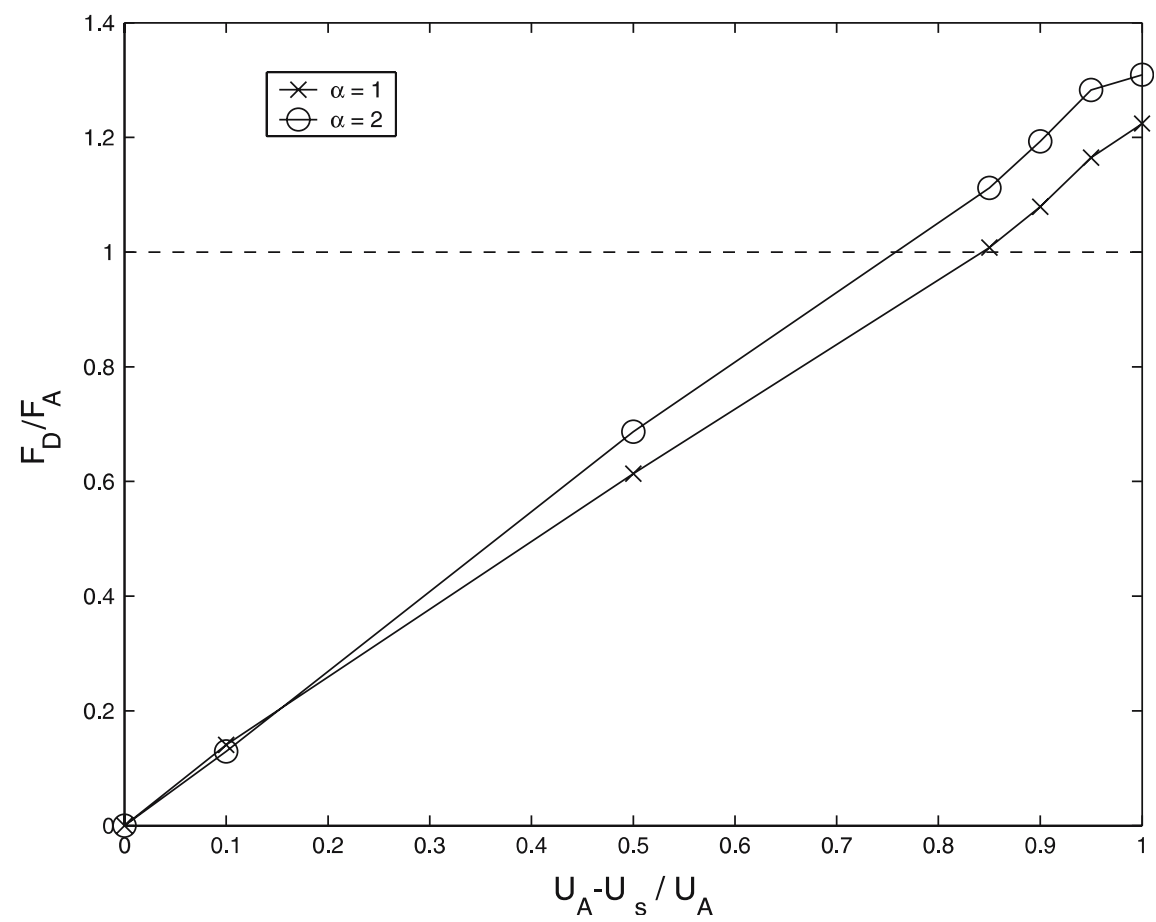

Figure 9. Influence of the fluid flow velocity generated by the acoustic wave $\left(U_{A}\right)$ and of the steady fluid flow velocity $\left(U_{S}\right)$ on the ratio between the hydrodynamic force and adhesion force for two values of the aspect ratio. Particle bridge removal will take place when this ratio is above 1 .

\subsection{INFLUENCE OF STEADY FLUID FLOW VELOCITY}

As mentioned earlier there is a steady fluid flow $\left(U_{S}\right)$ through the pore, which represents the oil flow rate through the reservoir during production. This steady flow pushes the particles against the pore throat. Therefore a larger hydrodynamic force from the acoustic wave is needed to remove the particle bridge than in the case that there is no steady flow. This effect is shown in Figure 9, where the ratio between the hydrodynamic force and adhesion force is shown as function of the ratio $\left(U_{A}-U_{S}\right) / U_{A}$. It is clear from this figure, that the effect of the steady flow is to reduce the cleaning possibility of the acoustic wave. A larger acoustic power input is needed for cleaning. This effect is very special for fouling due to particle bridge formation. It has also been found experimentally by Poesio and Ooms (2004).

\subsection{INFLUENCE OF ASPECT RATIO}

We define the aspect ratio as the ratio between the diameter of the narrow part of the pore and the particle diameter. The particles used in our 


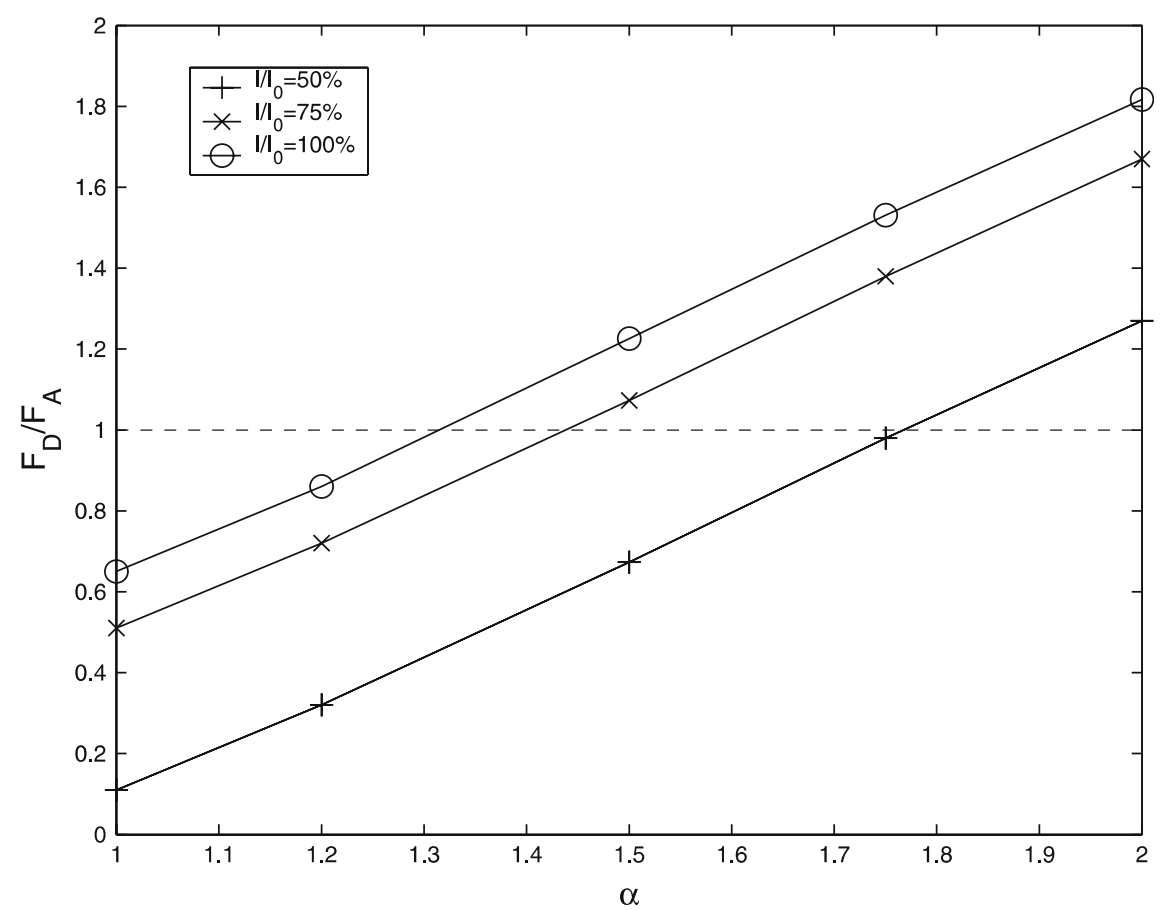

Figure 10. Influence of the aspect ratio $\alpha$ on the ratio between hydrodynamic force and adhesion force for three values of the acoustic input intensity $I$. $\left(I_{0}=P / S\right.$ is the reference intensity.) For values of $F_{D} / F_{A}$ larger than 1 , particle bridges are removed by the acoustic waves.

experiments have a rather narrow size distribution with a peak around $0.8-1.0 \mu \mathrm{m}$. The pore size distribution depends, of course, on the type of sandstone and can be rather broad. The values for the aspect ratio that we investigate in this publication, are between 1 and 2 . If the aspect ratio increases the hydrodynamic force on the particle bridge decreases. The reason is, that although the steady fluid flow velocity through the pore is kept constant the local velocity around the particles is smaller as the flow is less blocked at the entrance to the pore throat at larger values of the aspect ratio. The adherence force also decreases with increasing aspect ratio, because the average distance between the particles and the pore walls is larger. This last effect (decreasing adherence force) is considerably stronger than the first one (decreasing hydrodynamic force). So the net effect is, that with increasing aspect ratio the cleaning effect improves. This is shown in Figure 9, where the ratio between the hydrodynamic force and adhesion force is shown as function of the ratio $\left(U_{A}-U_{S}\right) / U_{A}$ for two values of the aspect ratio ( $\alpha=1$ and 2). In Figure 10 more details are given. In this figure the ratio between hydrodynamic force and adhesion force is shown as 
function of the aspect ratio (for three values of the relative acoustic input intensity $\left.I / I_{0}\right)$. An increase in aspect ratio at constant particle diameter means a larger pore diameter, which in its turn means a larger permeability of the porous material. So the conclusion is, that the cleaning efficiency improves with increasing permeability of the porous material.

\subsection{INFLUENCE OF ACOUSTIC FREQUENCY}

The acoustic frequency influences the hydrodynamic force in two ways. As the frequency increases the hydrodynamic force acting on the particles increases too, similarly to what happens to the drag force on a single particle exposed to an acoustic field in an unbounded medium, see Figure 2 and Equation (2). However, with increasing frequency also the damping of the high-frequency acoustic wave in a porous material increases, see Biot (1956). For a particle in the porous material at a certain distance (say $0.1 \mathrm{~m}$ ) from the acoustic source these two competing effects have to be taken into account. For low frequencies the particles bridge cannot be removed, as the hydrodynamic force is too small. For very high frequencies the particles bridge (at $0.1 \mathrm{~m}$ from the acoustic source) cannot be removed, because the acoustic wave has weakened too much due to the acoustic damping. The result can be seen in Figure 11 in which the ratio of the hydrodynamic force and the adhesion force is given as function of the (dimensionless) frequency for two values of the acoustic intensity and for two values of the aspect ratio. Only in a certain frequency interval particle bridge removal is possible. The frequency interval where acoustic cleaning is possible depends on parameters, such as acoustic input intensity and aspect ratio. The existence of a frequency interval for cleaning was found by Kuznetsov and Efimova (1983) during a field application. In their case the interval was between 5 and $50 \mathrm{kHz}$.

\section{Up-scaling of the Numerical Pore-level Results to the Macroscopic Permeability}

In order to compare our numerical results with experimental data an upscaling procedure based on a physical model for the porous material is developed. According to this model we assume that the porous material contains many pores with the shape sketched in Figure 12. Each pore is characterized by four parameters: $r_{1, i}, r_{2, i}, l_{1, i}$ and $l_{2, i}$ (see Figure 12). There are three types of such pores; each type is characterized by its own combination of $r_{1, i}, r_{2, i}, l_{1, i}$ and $l_{2, i}$ (so with $i=1,2$ or 3 ). So in each type of pore a pore throat is present for which the ratio of the throat diameter and the pore diameter is different. It can be shown, that the contribution of each 


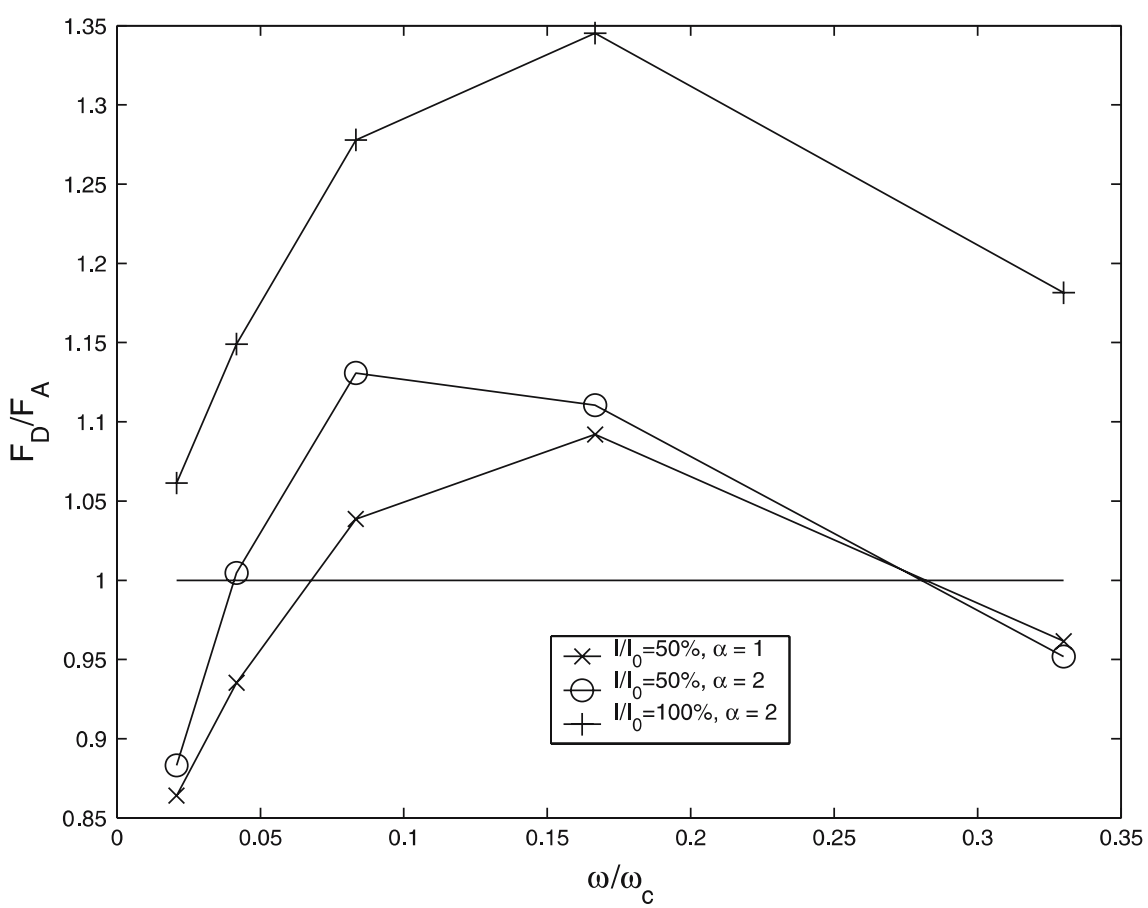

Figure 11. Influence of (dimensionless) acoustic frequency on the ratio between hydrodynamic force and adhesion force for two values of the (dimensionless) acoustic intensity $I / I_{0}$ and two values of the aspect ratio $\alpha$. (The frequency $\omega_{c}$ is the critical frequency from the Biot theory.) When the ratio $F_{D} / F_{A}$ is larger than 1 , particles bridge removal occurs.

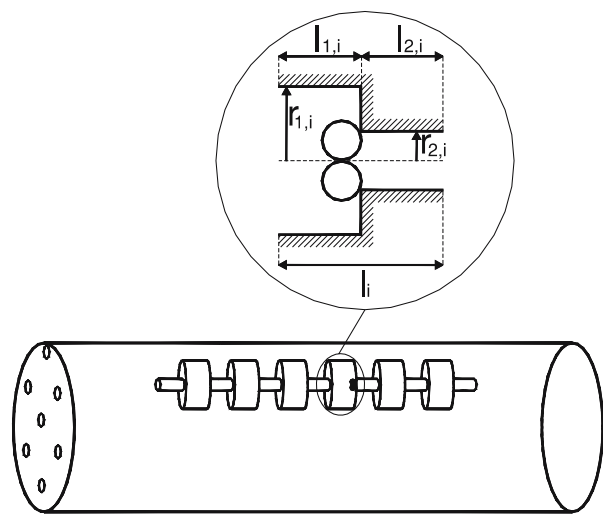

Figure 12. Conceptual model used for up-scaling of the numerical pore-level results to the macroscopic permeability. 
type of pores to the permeability is given by

$$
K_{i}=\frac{N_{i} \pi}{8}\left[\frac{l_{1, i}}{l} \frac{1}{r_{1, i}^{4}}+\frac{l_{2, i}}{l} \frac{1}{r_{2, i}^{4}}\right]^{-1},
$$

where $N_{i}$ is the number of pores of type $i$ per unit surface area in a perpendicular cross-section of the porous-material model, as sketched in Figure 12, and $l=l_{1}+l_{2}$. Equation 7 is explained in more detail by Kelder (1998). The total permeability $K$ is calculated from the contributions of the three types of pores in the following way

$$
\frac{1}{K}=\sum_{i} \frac{1}{K_{i}}
$$

This procedure to calculate the effective (up-scaled) permeability is rather approximate. Harmonic averaging is correct only if the flow channel is exactly one-dimensional. In a porous medium with three-dimensional channels there is a possibility of by-pass flow which causes a higher effective $K$. It is possible that the discrepancy between experiments and theory (to be discussed) is caused by this too approximate up-scaling.

The next step is to introduce fouling by particles into the model. This is achieved by assuming a certain number of pores to be blocked by particle bridges. This fraction can be different for the three pore types. So in the fouled area the numbers $N_{1}, N_{2}$ and $N_{3}$ are different from their original values for the clean porous material. The fouling is supposed to be uniformly distributed in the porous material. Using Equation 7 we can calculate the permeability for the fouled porous material.

The cleaning of the material by acoustic waves is then calculated at the pore level using the procedure explained earlier. For certain values of the relevant parameters (the acoustic intensity, frequency, steady fluid flow velocity, aspect ratio) the hydrodynamic force $F_{D}$ is calculated as function of the distance from the acoustic source. A typical result is shown in Figure 13. Where $F_{D}$ is larger than the adhesion force $F_{a}$ the porous material will be cleaned. In the other part the particle bridges are not removed by the high-frequency acoustic waves. As can be seen a typical value for the cleaned area is $0.1-0.2 \mathrm{~m}$ from the acoustic source. After this pore level calculation we can use Equation 7 again the determine the permeability distribution after the cleaning process.

As mentioned earlier the cleaning process is due, mainly, to the fast acoustic waves. Shear waves are assumed to be absent because of the perpendicular incidence of the waves onto the material. The slow wave is damped very quickly; it penetrates only a few millimeters. The fast wave can penetrate a larger distance. Dependent on the frequency it is of the order of $0.2 \mathrm{~m}$. In earlier work we reported about measurements of the damping coefficient (Poesio, Ooms, Schraven and Van der Bas, 2002). 


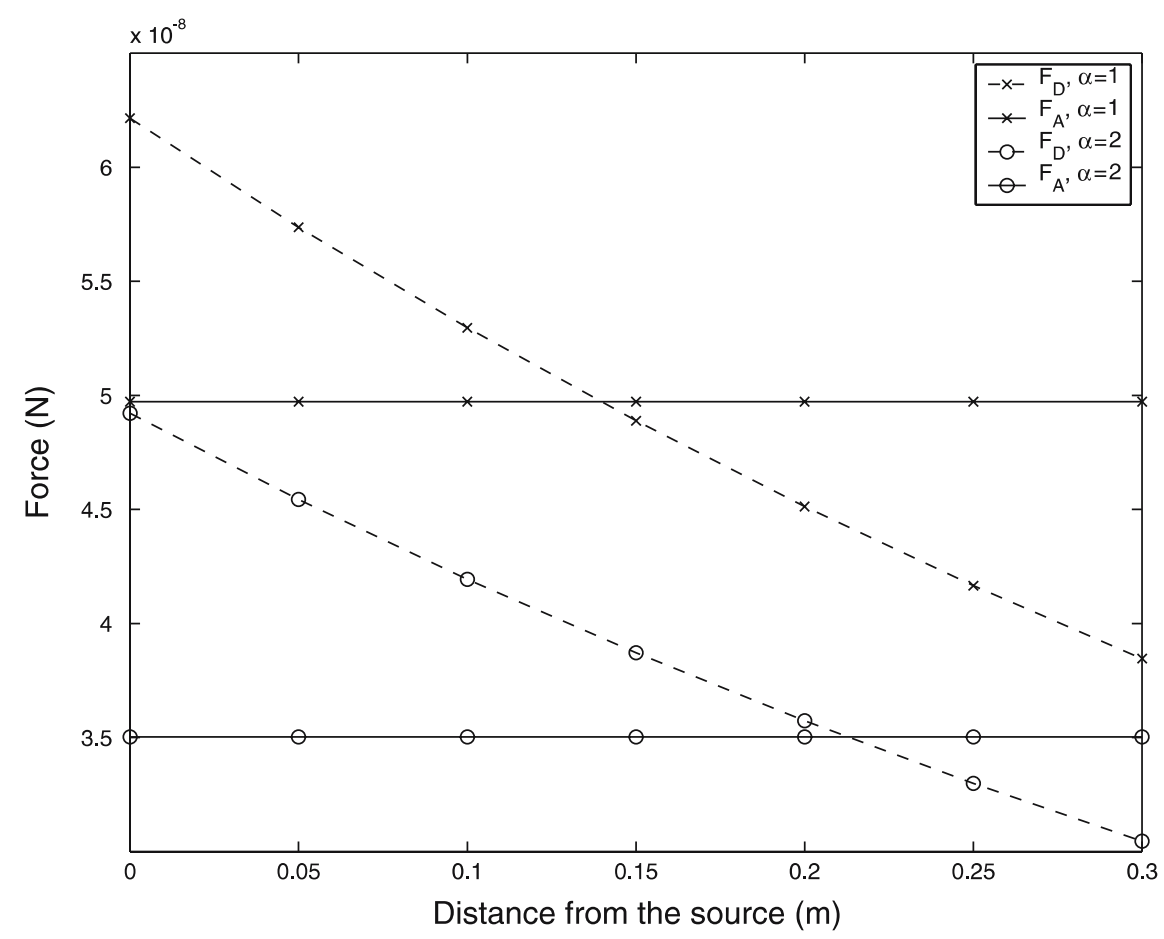

Figure 13. Comparison between colloidal adhesion force and hydrodynamic detaching force as function of the distance form the acoustic source.

\section{Comparison with Experiments}

We will now use the calculation procedure for the permeability improvement due to ultrasonic cleaning (as described in the foregoing paragraph) to compare permeability data obtained from cleaning experiments on Berea sandstone cores with their numerical predictions. The experiments have been described in detail by Poesio and Ooms (2004). We will not repeat these details here. We will only use some of the experimental results to check the reliability of the numerical calculation procedure for the permeability improvement.

Using the known properties of the porous material and of the acoustic waves used during the experiments, the cleaning efficiency can be calculated and compared with the experimental data. During the experiments measurements were carried out over three parts of the sandstone cores: the first part (closest to the acoustic source), the second part (in the middle of the core) and the third part (furthest away from the acoustic source). The first part has a length of about $0.025 \mathrm{~m}$. The total core length is about $0.2 \mathrm{~m}$. It is clear that the cleaning process is in particular successful in the first part of the core, where the damping effect on the acoustic wave is 

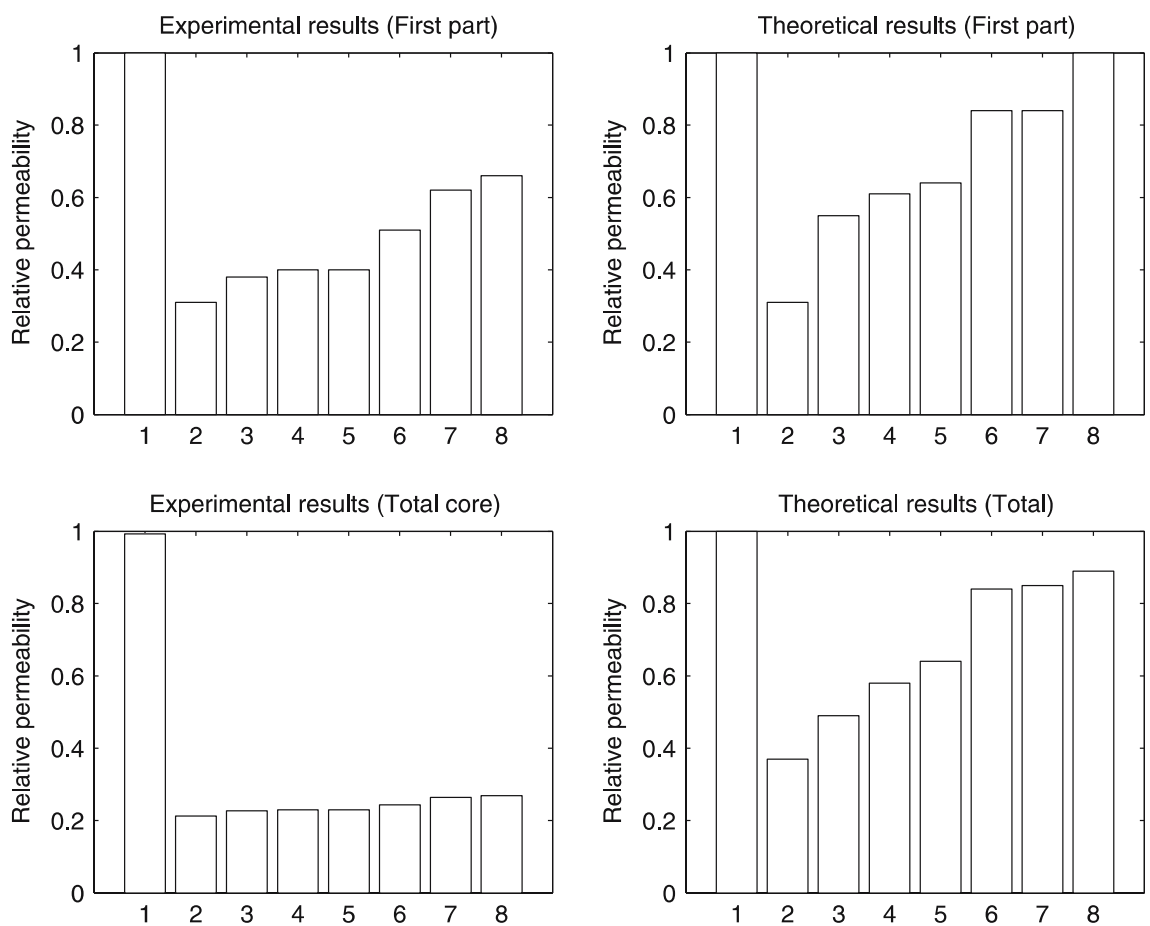

Figure 14. Comparison between experimental cleaning results and their model predictions. Step 1 gives the value of the relative permeability before fouling and step 2 the value after fouling. Steps 3, 4 and 5 show the relative permeability improvement due to an increase of the acoustic amplitude. Steps 6, 7 and 8 show the relative permeability improvement due to a decrease in net fluid flow rate. For more details see Table I.

Table I. Experimental conditions used at each stage of the test

\begin{tabular}{llllll}
\hline $\begin{array}{l}\text { Treatment } \\
\text { step }\end{array}$ & Description & Power $(\mathrm{W})$ & $\begin{array}{l}\text { Pressure } \\
(\mathrm{MPa})\end{array}$ & $\begin{array}{l}\text { Temperature } \\
\left({ }^{\circ} \mathrm{C}\right)\end{array}$ & $\begin{array}{l}\text { Flow } \\
\text { rate }(\mathrm{ml} / \mathrm{min})\end{array}$ \\
\hline 1 & Start & - & 12 & 20 & - \\
2 & Fouling & - & 12 & 20 & 25 \\
3 & Back flow & - & 12 & 20 & 5 \\
4 & Acoustic treatment & 350 & 12 & 20 & 75 \\
5 & Acoustic treatment & 500 & 12 & 20 & 75 \\
6 & Acoustic treatment & 700 & 12 & 20 & 75 \\
7 & Acoustic treatment & 700 & 12 & 20 & 5 \\
8 & Acoustic treatment & 700 & 12 & 20 & 0 \\
\hline
\end{tabular}



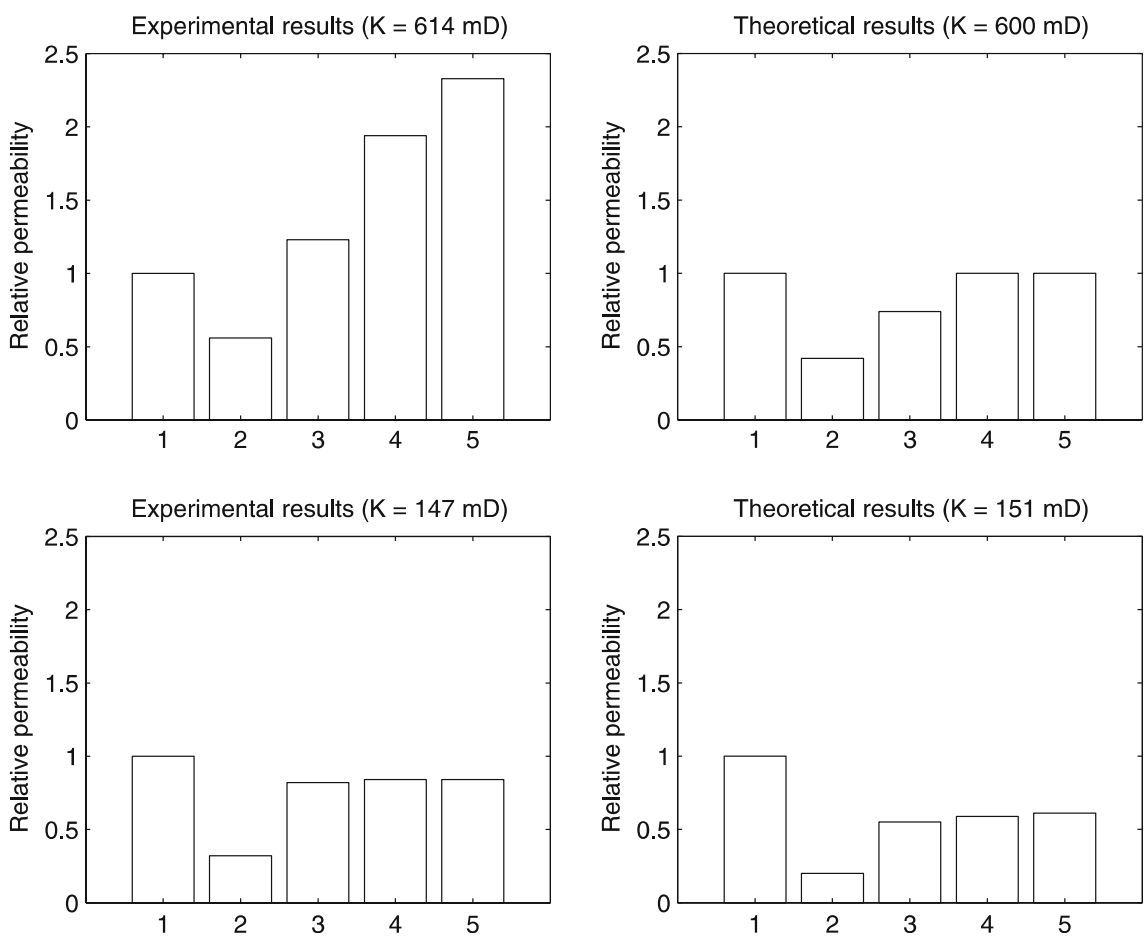

Figure 15. Comparison between experimental cleaning results and their model predictions for two values of the initial permeability of the porous material. Step 1 gives the value of the relative permeability before fouling and step 2 the value after fouling. Steps 3, 4 and 5 show the relative permeability improvement due to an increase of the acoustic amplitude. For more details see Table II.

not yet very strong. In Figures 14 and 15 we present results for the first part and for the total core (part 1, 2 and 3 together, see Poesio and Ooms, 2004). In these figures the relative permeability is defined as the ratio of the permeability during the cleaning process and the initial permeability of the cores before fouling. Step 1 gives the value of the relative permeability before fouling and is by definition equal to 1 . Step 2 gives the permeability after fouling of the core. The fouling procedure for the experiments is described in the publication by Poesio and Ooms, (2004) and is, therefore, not repeated here. The fouling procedure for the numerical simulations has been described in the preceding section. Numerical fouling is achieved by assuming a certain number of pores to be blocked by particle bridges. As mentioned this fraction can be different for the three pore types. In this way we adapt the initial fouling for the numerical simulations to the initial fouling level of the experiments.

In Figure 14 the influence of the acoustic amplitude and of the steady fluid flow rate on the cleaning efficiency is shown. Steps 3, 4 and 5 show 
Table II. Experimental conditions used at each stage of the test

\begin{tabular}{llllll}
\hline $\begin{array}{l}\text { Treatment } \\
\text { step }\end{array}$ & Description & $\begin{array}{l}\text { Power } \\
(\mathrm{KW})\end{array}$ & $\begin{array}{l}\text { Pressure } \\
(\mathrm{Mpa})\end{array}$ & $\begin{array}{l}\text { Temperature } \\
\left({ }^{\circ} \mathrm{C}\right)\end{array}$ & Flow rate $(\mathrm{ml} / \mathrm{min})$ \\
\hline 1 & Start fouling & - & 12 & 20 & - \\
2 & Back flow & - & 12 & 20 & 25 \\
3 & Acoustic treatment & 1 & 12 & 20 & 5 \\
4 & Acoustic treatment & 1.5 & 12 & 20 & 5 \\
5 & Acoustic treatment & 2 & 12 & 20 & 5 \\
\hline
\end{tabular}

the relative permeability improvement due to an increase of the acoustic amplitude. Steps 6, 7 and 8 show the relative permeability improvement due to a decrease in net fluid flow rate. The core cleaning improves with increasing amplitude as the acoustic waves can penetrate deeper into the core material, before they are damped because of their interaction with the porous material. The cleaning improves with decreasing steady fluid flow rate, as the particles bridges can more easily be removed from the pore throat. The agreement between experimental data and model predictions is reasonable for the first part of the core. However, for the total core the cleaning efficiency is over-predicted by the model. A possible explanation is, that according to our model detached particles do not contribute to the fouling of the material anymore. However, it is possible that such particles are involved in the formation of new particle bridges. This effect is not taken into account in the model. Even when the detached particles are not involved in the formation of new particle bridges, they can significantly influence the fluid flow through the porous material. Also this effect is not taken into consideration. Moreover our calculations are based on the assumption, that a particle bridge consists of two particles. In reality such bridges can contain many particles and can even consist of a number of particle layers, which could strengthen the particle bridge. Finally, perhaps the application of non-linear acoustic theory would yield better predictions. It is clear, that much research needs to be done.

The effect of the absolute value of the initial permeability of the core material on the cleaning efficiency can be seen from Figure 15. Experiments on cores with a permeability of 614 and $147 \mathrm{mD}$ have been carried out. Step 1 gives again the value of the relative permeability before fouling and step 2 the value after fouling. Steps 3, 4 and 5 show the relative permeability improvement due to an increase of the acoustic amplitude. As can be seen the cleaning efficiency improves with increasing initial permeability of the porous material. Also the beneficial effect of a larger acoustic amplitude on the cleaning is again 
observed. The agreement between experimental results and model predictions is only qualitatively reasonable. A peculiar effect can be seen for the experiments on cores with a permeability of $614 \mathrm{mD}$. The relative permeability of the material can become larger than 1 during the cleaning process. This phenomenon can, of course, not be explained by our model. It is likely caused by a disintegration of the sandstone due to the acoustic waves.

\section{Conclusions}

We have studied the possibility of removing particle bridges from a porous material by high-frequency acoustic waves. To that purpose we have developed a numerical calculation procedure (based on the lattice-Boltzmann method) to determine the hydrodynamic force on the particles in a bridge (consisting of two particles) blocking a pore throat, and we have compared the hydrodynamic force with the adhesion force between the particles and the pore throat material. (We emphasize, that the calculation does not continue after the detachment of the particle from the pore wall. Once the hydrodynamic force becomes larger than the adhesion force the calculation is stopped.) From this comparison follows, whether a particle bridge is removed from the pore throat, or not. The sensitivity of several relevant parameters, such as acoustic wave amplitude, acoustic frequency, aspect ratio and steady fluid flow velocity has been investigated. A calculation procedure has been developed to up-scale the numerical pore level results to the macroscopic permeability. We have used this procedure to compare numerical predictions for the efficiency of the acoustic cleaning technique with experimental data derived from ultrasonic cleaning experiments on Berea sandstone cores. The agreement is only qualitatively reasonable.

\section{References}

Biot, M. A.: 1956, Theory of propagation of elastic waves in a fluid-saturated porous medium. Low frequency range, J. Acoust. Soc. Am. 28, 168-191.

Ten Cate, A.: 2002, Turbulence and particle dynamics in dense crystal slurries, $\mathrm{PhD}$ Thesis, Delft University of Technology.

Cherukat, P. and McLaughlin, J. B.: 1994, The inertial lift on a rigid sphere in a linear shear flow field near a flat wall, J. Fluid Mech. 263, 1-18.

Derksen, J. J. and Van den Akker, H. E. A.: 1999, Large Eddy simulations on the flow by a rushton turbine, AICHE Journal 45, 209-221.

Eggels, J. M. G. and Somers, J. A.: 1995, Numerical simulation of free convective flow using lattice-Boltzmann scheme, Int. J. Heat Fluid Flow 16, 357-364.

Hasimoto, H.: 1959, On the periodic fundamental solutions of the Stokes equations and their application to viscous flow past a cubic array of spheres, J. Fluid Mech. 5, 317-328. 
Kelder, O.: 1998, Frequency-dependent wave propagation in water-saturated porous media, PhD Thesis, Delft University of Technology.

Khilar, K. C. and Fogler, H. S.: 1998, Migration of fines in porous media, Kluwer Academic Publishers.

Kuznetsov, O. L. and Efimova, S. A.: 1983, Application of Ultrasound in Oil Industry, Nedra Press (in Russian).

Ladd, A. J. C.: 1994a, Numerical simulation of particulate suspensions via a discretized Boltzmann equation. Part 1: Theoretical foundation, J. Fluid Mech. 271, 271-309.

Ladd, A. J. C.: 1994b, Numerical simulation of particulate suspensions via a discretized Boltzmann equation. Part 2: Numerical Results, J. Fluid Mech. 271, 311-339.

Landau, L. D. and Lifshitz, E. M.: 1959, Fluid Mechanics, Pergamon Press.

Poesio, P. and Ooms, G.: 2004, Formation and ultrasonic removal of fouling particles structures in a natural porous material, J. Petroleum Sci. Eng. 45, 159-178.

Poesio, P., Ooms, G., Barake, S. and Van der Bas, F.: 2002, An investigation of the influence of acoustic waves on the liquid flow through a porous material, J. Acoust. Soc. Am. 111, 2019-2025.

Poesio, P., Ooms, G., Schraven, A. and Van der Bas, F.: 2002, Theoretical and experimental investigation of acoustic streaming in a porous material, Phys. Rev. E 66, article number 016309.

Poesio, P., Ooms, G., Van Dongen, M. E. H. and Smeulders, D. M. J.: 2004, Removal of small particles from a porous material by ultrasonic irradiation, Trans. Porous Media 54, 239-264. 